\title{
Thermal Noise Measurements in a Reverberation Chamber
}

This paper was downloaded from TechRxiv (https://www.techrxiv.org).

\section{LICENSE}

CC BY 4.0

SUBMISSION DATE / POSTED DATE

$11-01-2022$ / 14-01-2022

CITATION

Marvin, Andy; Bale, Simon J (2022): Thermal Noise Measurements in a Reverberation Chamber. TechRxiv. Preprint. https://doi.org/10.36227/techrxiv.18134063.v1

$\mathrm{DOI}$

10.36227/techrxiv.18134063.v1 


\title{
Thermal Noise Measurements in a Reverberation Chamber
}

\author{
Andrew C. Marvin, Life Fellow, IEEE, and Simon J. Bale
}

\begin{abstract}
Two models describing properties of the thermal noise power emerging from an antenna mounted in a reverberation chamber are described. The first compares the reverberation chamber to an over-moded cavity acting as a blackbody source; the second describes the noise in terms of the antenna noise temperature. Measurements based on the antenna noise temperature model show the noise power to be dependent on the position of the rotating mechanical stirrer. The correlation of the antenna noise temperature with the stirrer position dependent antenna mis-match factor is shown. When a signal is injected into the chamber measurements of the signal to noise power ratio show that this ratio is dependent on the stirrer position and also the position of the signal source within the chamber. These results have significant consequences for the detection of weak signals from radiating sources in a reverberation chamber.
\end{abstract}

Index Terms - reverberation chamber, antenna noise temperature, signal-to-noise ratio.

\section{INTRODUCTION}

$\mathrm{T}$ HIS letter describes experiments undertaken to examine the thermal noise properties of an electromagnetic reverberation chamber, in particular the noise power emergent from a receiving antenna installed in the chamber. Reverberation chamber measurements are well established for EMC using international measurement standards such as IEC 61000-4-21 [1] for EMC measurements or IEEE Standard 2991 [2] for Shielding Effectiveness measurements of enclosures. Radio system measurements include antenna parameters, for example antenna efficiency [3] and radio system performance [4]. All reverberation chamber measurements require the use of antennas in the chamber to inject or to receive signals. As with any antenna-based measurement system, the minimum signal level that can be detected depends on the noise performance of the receiver and on the thermal noise received by the antenna from its surroundings in the chamber.

In this letter we examine the thermal noise received by an antenna in a reverberation chamber showing that the received noise is variable and dependent on the position of the mechanical stirrer. In II two theoretical models of noise in a reverberation chamber are described. The antenna noise measurement technique is described in III and the results of the noise measurements are shown and their correlation with the antenna mis-match factor is demonstrated. In IV the power

Manuscript received date.

The authors are with the Department of Electronic Engineering, University of York, York, UK (email andy.marvin@york.ac.uk, simon.bale@york.ac.uk ). received from a signal source in the chamber is examined and shown to depend on the position of the source as well as the stirrer position. The signal-to-noise ratio consequences of the noise power variability and antenna position are demonstrated.

\section{THEORETICAL MODELS FOR THERMAL NOISE IN A REVERBERATION CHAMBER}

\section{A. Cavity with an Aperture Model}

Thermal radiation is emitted by any physical body with a temperature above absolute zero. Plank's Law determines the radiated noise power in a given frequency interval as a function of frequency and temperature for a "black body" having a surface emissivity of unity. For a partially reflective body, the radiated noise power is reduced by the surface emissivity which is less than unity. At radio frequencies, and for surface temperatures in the region of $300 \mathrm{~K}$, the Rayleigh-Jeans approximation to Plank's Law can be used indicating that the radiated power in a frequency interval is directly proportional to the absolute temperature of the body. Many texts which describe black body radiation, for example [5] and [6], describe a type of black body source comprising a cavity large compared to the wavelength, supporting many resonant modes, with an aperture small compared to the cavity dimensions but large compared to the wavelength. A ray entering the cavity undergoes multiple imperfect reflections and is considered unlikely to emerge from the aperture before its power is exhausted. This means that the aperture is effectively a black body absorber. Kirchhoff's Law of thermal radiation states that the absorption and emission properties of the system are equal at thermal equilibrium. If the cavity is held at a fixed temperature the energy propagating inside is in thermal equilibrium with the cavity. The small aperture has a negligible effect on this equilibrium although some energy will escape. The cavity acts as a black body radiator with its emitted spectrum determined by Plank's Law and the surface temperature of the cavity. This type of cavity forms the basis of many commercial black body sources sold for laboratory use.

A reverberation chamber with a receiving antenna operated at radio frequencies can be considered to be a low frequency implementation of such a cavity. The chamber walls and the stirrer are imperfect reflectors. The small aperture is replaced by the antenna's receiving aperture. The implementation is not 
exact as the antenna replacing the aperture has a receiving aperture with dimensions comparable to the wavelength. As the reverberation chamber is stirred the imperfections in its "black body" characteristics will be a perturbed by changes of the stirrer position. This effect is demonstrated in Section III $B$ below.

\section{B. Antenna Noise Temperature Model}

From a radio frequency measurement perspective, a more approachable model of antenna thermal noise in a reverberation chamber is the Antenna Noise Temperature. The thermal noise received by the antenna is represented by a noise source embedded in the real part of the antenna radiation impedance in the same way that thermal (Nyquist) noise is represented in a resistor. The total noise power present at the input to the receiver under conjugate impedance match conditions between the antenna and the receiver is then $P_{\text {noise }}$;

$$
P_{\text {noise }}=\left(T_{\text {ant }}+T_{\text {sys }}\right) k B
$$

where $T_{\text {ant }}$ is the equivalent antenna noise temperature, $T_{s y s}$ is the receiving system noise temperature referred to its input, $k$ is Boltzmann's constant and $B$ is the receiving system measurement bandwidth. Note that $T_{\text {sys }}$ is related to the more common receiving system input Noise Figure, $N F$ by;

$$
N F=10 \log _{10}\left(1+T_{\text {sys }} / 290\right) d B
$$

where $290 \mathrm{~K}$ is the standard noise source noise temperature.

In a perfect anechoic chamber with a conjugate impedance match between the antenna and the receiver $T_{\text {ant }}$ is the ambient temperature of the chamber walls. In a reverberation chamber the reverberant backscatter will modify the antenna - receiver impedance match as a function of the stirrer position and thus the apparent antenna noise temperature.

\section{ANTENNA NoISE TEMPERATURE MEASUREMENT IN THE REVERBERATION CHAMBER}

\section{A. Antenna Noise Temperature Formulation}

In this section the formulation used to derive the antenna noise temperature in the reverberation chamber is derived. The formulation takes the form of a comparison of the noise temperature of the antenna with that of a $50 \Omega$ load at the same ambient temperature as the antenna and chamber and connected to the input of the receiver.

Under impedance matched conditions the total noise power referred to the input of a receiver is given by (1) above. In terms of power, the total receiver input noise power with either an antenna or a $50 \Omega$ load at the receiver input is;

$$
\left\{\begin{array}{l}
P_{\text {NoiseANT }}=P_{A N T}+P_{\text {sys }} \\
P_{\text {Noise } 50 \Omega}=P_{50 \Omega}+P_{\text {sys }}
\end{array}\right.
$$

where $P_{A N T}$ is the noise power from the antenna, $P_{50 \Omega}$ is the noise power from the $50 \Omega$ load and $P_{\text {sys }}$ is the equivalent input noise power of the receiver related to its input Noise
Temperature. The quantities $P_{\text {NoiseANT }}$ and $P_{\text {Noise50S }}$ are the measured quantities. Noting that;

$$
\frac{P_{50 \Omega}}{T_{50 \Omega}}=k B=\frac{P_{s y s}}{T_{s y s}} \quad P_{s y s}=P_{50 \Omega} \cdot \frac{T_{s y s}}{T_{50 \Omega}}
$$

and combining with (3) gives,

$$
\begin{gathered}
P_{50 \Omega}=\frac{P_{\text {Noise } 50 \Omega}}{1+{ }^{T_{\text {Sys }}} / T_{50 \Omega}} \quad P_{A N T}=\frac{P_{\text {NoiseANT }}}{1+{ }^{T} A N T / T_{50 \Omega}} \\
\frac{P_{A N T}}{P_{50 \Omega}}=\frac{T_{A N T}}{T_{50 \Omega}}=\frac{P_{\text {Noise } A N T}}{P_{\text {Noise } 50 \Omega}} \cdot \frac{1+T_{\text {sys }} / T_{50 \Omega}}{1+T_{\text {sys }} / T_{A N T}} \\
T_{A N T}=\frac{P_{\text {NoiseANT }}}{P_{\text {Noise } 50 \Omega}} \cdot\left(T_{50 \Omega}+T_{\text {sys }}\right)-T_{\text {sys }}
\end{gathered}
$$

The antenna noise temperature can be calculated from the

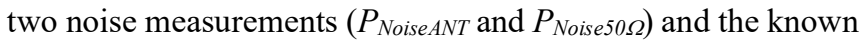
values of the temperature of the $50 \Omega$ load and the receiver system noise temperature.

In the case of an antenna and receiver that do not have a conjugate impedance match (6) requires modification to account for the mis-match factors. The input scattering parameters of the antenna and receiver can be measured as $S 11_{A N T}$ and $S 11_{s y s}$. The mis-match correction factor derived from [7] is then

$$
\frac{\left(1-\left|S 11_{A N T}\right|^{2}\right)\left(1-\left|S 11_{s y s}\right|^{2}\right)}{\left|1-S 11_{A N T} S 11_{s y s}\right|^{2}}
$$

The equivalent correction factor for the receiver system to load mismatch is derived by replacing $S 11_{A N T}$ with $S 11_{50 \Omega}$. Because the $S 11_{50 \Omega}$ of the load is zero when measured with a $50 \Omega$ characteristic impedance network analyser system, the correction factor reduces to

$$
\left(1-\left|S 11_{\text {sys }}\right|^{2}\right)
$$

The overall mis-match correction ratio $C$ for the ratio of $P_{\text {Noise }}$ ${ }_{A N T}$ to $P_{\text {Noise } 50 \Omega}$ is the ratio of the two factors above.

$$
C=\frac{\left(1-\left|S 11_{A N T}\right|^{2}\right)}{\left|1-S 11_{A N T} S 11_{s y s}\right|^{2}}
$$

\section{B. Antenna Noise Temperature Measurements}

The reverberation chamber at the University of York has dimensions of $4.7 \mathrm{~m} \times 3.0 \mathrm{~m} \times 2.4 \mathrm{~m}$. All measurements were made at a frequency of $800 \mathrm{MHz}$, the optimum frequency for lowest noise of the receiver system.

Fig. 1 shows a block diagram of the measurement system. The receiving antenna is mounted on the chamber wall and the measurement reference plane is the wall mounted coaxial connector adjacent to the antenna. The antenna $S 11_{A N T}$ is measured by connecting the network analyser at the reference plane. The noise power emerging from the antenna $P_{A N T}$ along with the equivalent system input noise power $P_{s y s}$ is measured 
by connecting the signal analyser at the reference plane, the measurand being $P_{\text {NoiseANT. }}$

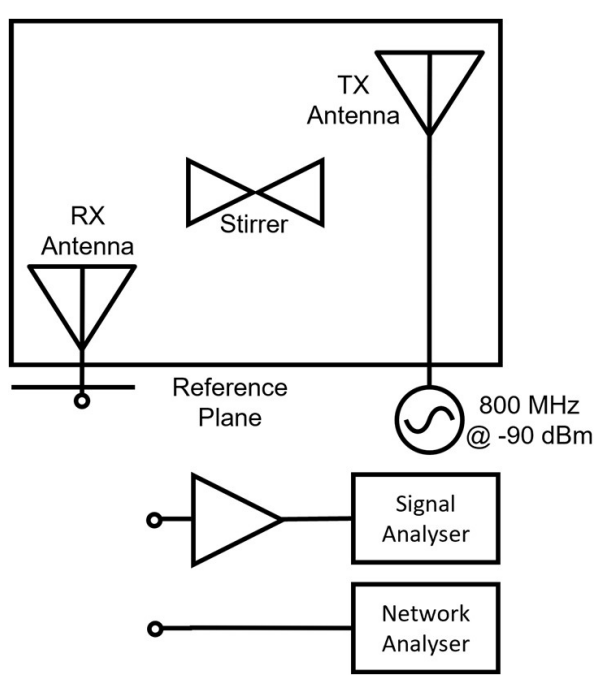

Fig. 1. Block diagram of the reverberation chamber measurement set-up. The parameters of the amplifier at the input to the signal analyser are described in the text below.

The transmitting antenna is used to inject a signal into the chamber for the signal-to-noise measurements detailed in Section IV below. These antennas are identical and described in [8]. The receiver system comprises a pre-amplifier comprising a cascaded pair of Mini-Circuits ZX60-1215LN amplifiers each with a specified maximum Noise Figure of 0.4 $\mathrm{dB}$ (equivalent to a maximum input noise temperature of $28 \mathrm{~K}$ ) and an overall gain of $34 \mathrm{~dB}$ at $800 \mathrm{MHz}$. This is followed by an HP8447D intermediate amplifier with a gain of $26 \mathrm{~dB}$ and a Noise Figure of $8 \mathrm{~dB}$ feeding an Agilent EXA Signal Analyser. The overall system noise temperature, $T_{\text {sys, }}$ measured at the preamplifier input port using a HP8970B Noise Figure Meter is 34 K (0.48 dB Noise Figure).

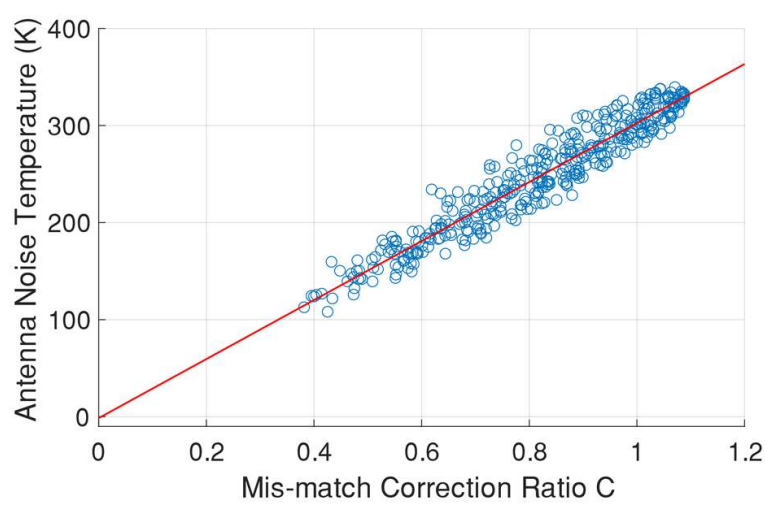

Fig. 2. Correlation of the measured antenna noise temperature of the wall mounted antenna in position two and the mis-match correction ratio, $\mathrm{C}$.

The $\mathrm{S}$ parameters used for the mis-match correction factor estimates were measured using a Rhode and Schwartz ZVB20 vector network analyser.

For the noise power measurements, a measurement bandwidth of $10 \mathrm{kHz}$ was used. This bandwidth was chosen after examination of the frequency dependence of $S 11_{A N T}$. A wider bandwidth would have required correction for the frequency dependence of $S 11_{A N T}$. Narrower bandwidths result in enhanced signal to noise ratios in the normal way but at the expense of increased measurement time. The signal analyser records 1001 noise power measurements in one time sweep at $800 \mathrm{MHz}$. These are averaged to give $P_{A N T}$ at each stirrer position and also $P_{50 \Omega}$.

Fig. 2 shows the measured antenna noise temperatures calculated according to equation (6) compared with the mismatch correction ratios derived from (7) at each of 400 stirrer positions within one rotation of the stirrer. Clearly there is a strong linear correlation, a Pearson correlation coefficient [9] of $\rho=0.96$ was calculated. The least squares regression line is shown in red, this intersects the temperature axis at $-1.4 \mathrm{~K}$. Note that at a minority of stirrer positions the mis-match correction ratio is greater than one. This occurs because the receiver system has an input impedance that is not $50 \Omega$. At some stirrer positions the antenna output impedance offers a better impedance match to the receiver than the $50 \Omega$ load. The temperature value of the of the best-fit line shown in Fig. 3 for a mis-match correction ratio value of unity shows the estimated antenna noise temperature corrected for the mis-match factor is equal to the ambient temperature of the chamber, in this case $302 \mathrm{~K}$. A thermocouple from an electronic thermometer was bonded to the chamber wall during the measurements. For the data shown in Fig. 2 the thermometer recorded a temperature of $301 \mathrm{~K}$. This result confirms the perturbed cavity black body source description of the reverberation chamber detailed in Section II $A$.

\section{SIGNAL-TO-NOISE RATIO MEASUREMENTS IN THE REVERBERATION CHAMBER}

The transmitting antenna shown in Fig. 1 was used to inject a $\mathrm{CW}$ signal into the reverberation chamber with a

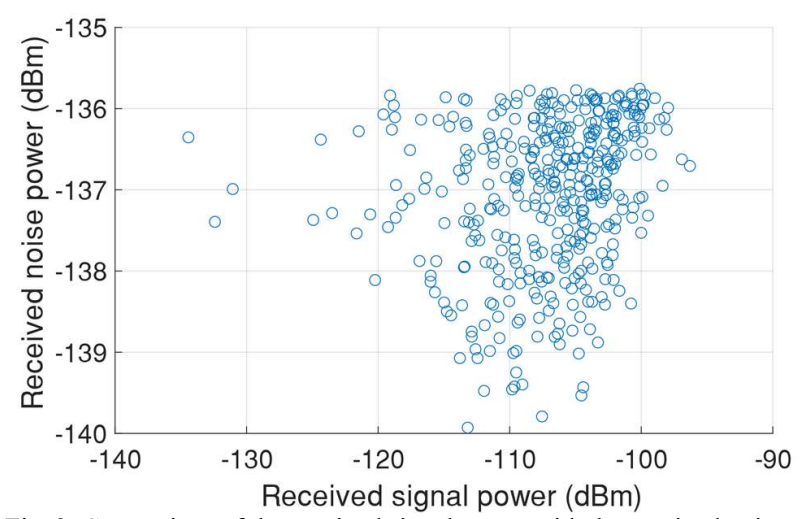

Fig. 3. Comparison of the received signal power with the received noise power in a $10 \mathrm{kHz}$ bandwidth at each stirrer position for the wall mounted antenna in position two. $60 \mathrm{~dB}$ was subtracted from the measured values to account for the receiver system gain.

forward power of $-90 \mathrm{dBm}$ at the same 400 stirrer positions as the noise measurements.

The received signal power at the noise temperature measurement antenna was used to measure the received $\mathrm{CW}$ power to estimate the signal-to-noise ratio at each stirrer position. It can be seen in Fig. 3 that there is almost no correlation between the total noise power, $P_{\text {NoiseANT }}$ in (3), and 
the signal power at each stirrer position, a Pearson correlation coefficient of $\rho=0.27$ was calculated. We believe that the very weak correlation here is due to the difference in the energy propagation paths between the antenna-to-antenna signal propagation and the distributed chamber noise source to antenna propagation.

For comparison, the results of a second set of identical noise power, $S 11_{A N T}$ and $\mathrm{CW}$ signal power measurements are shown with the signal injection antenna moved from its original position a distance of $375 \mathrm{~mm}$ (one wavelength at $800 \mathrm{MHz}$ ).
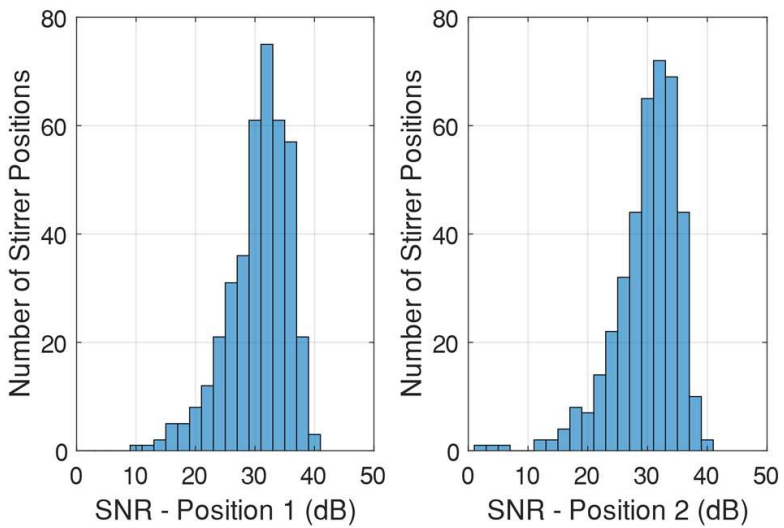

Fig. 4. Histograms of the signal-to-noise ratios at each of the 400 stirrer positions for signal the original injection antenna position (position 1) and the second position 2 .

Histograms of the signal-to-noise ratios for the two antenna positions are shown in Fig. 4. Comparison of these two data sets using the two-sample Kolmogorov-Smirnov test implemented in Matlab [10] indicates that the two data sets have the same statistical distribution. Whilst the two populations have the same distribution, the individual SNR values at each stirrer position differ between the two populations.

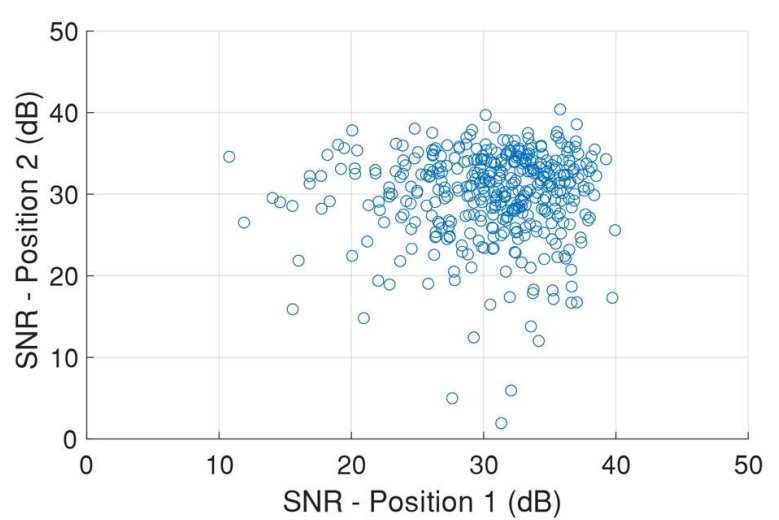

Fig. 5. Scatter plot showing the signal-to-noise ratios for the two signal injection antenna positions. A Pearson correlation coefficient of 0.024 was calculated indicating a lack of linear correlation.

In Fig. 5, the scatter plot reveals that the optimum signal-tonoise ratio is found at different stirrer positions for the two transmitting antenna positions. Therefore, the optimum detection of weak signals in the presence of noise in a reverberation chamber requires examination of each stirrer position

\section{CONCLUSION}

In this letter we have demonstrated that a reverberation chamber exports thermal noise power through its receiving antenna at a level proportional to the ambient temperature of the chamber walls and to the impedance mis-match correction factor between the antenna and the receiving system at each stirrer position. This leads to a population of apparent antenna noise temperatures, one for each of the stirrer positions. Measurements of the received signal power level from a source radiating into the chamber are only weakly correlated with the noise power level received at each stirrer position. This leads to a population of signal to noise ratios. Changing the position of the signal source in the chamber results in a different population of signal to noise power ratios. While each population has the same statistical distribution, the individual samples in the population measured at each stirrer position have different values. The optimum signal to noise ratios are at different stirrer positions. Thus, the detection of weak signals in a reverberation chamber has to be done for a particular equipment configuration in the chamber by examining each stirrer position.

\section{REFERENCES}

[1] International Electrotechnical Commission, 61000-4-21 ELECTROMAGNETIC COMPATIBILITY (EMC), Part 4: Testing and Measurement Techniques, Section 21: Reverberation Chamber Test Methods.

[2] "IEEE Standard Method for Measuring the Shielding Effectiveness of Enclosures and Boxes Having all Dimensions between $0.1 \mathrm{~m}$ and $2 \mathrm{~m}$," in IEEE Std 299.1-2013, vol., no., pp.1-96, 15 Jan. 2014, doi: 10.1109/IEEESTD.2014.6712029.

[3] C. L. Holloway, H. A. Shah, R. Pirkl, W. F. Young, D. A. Hill and J. Ladbury, "A three-antenna technique for determining the total and radiation efficiencies of antennas in reverberation chambers," in IEEE Antennas and Propagation Magazine, vol. 54, no. 1, pp. 235-241, Feb. 2012, doi: 10.1109/MAP.2012.6209518.

[4] C. L. Holloway, D. A. Hill, J. M. Ladbury, P. Wilson, G. Koepke, and J. Coder, "On the use of reverberation chambers to simulate a controllable Rician radio environment for the testing of wireless devices," IEEE Trans. Antennas Propag., Special Issue on Wireless Communications, vol. 54, no. 11, pp. 3167-3177, Nov. 2006.

[5] G.F.C. Rogers and Y.R. Mayhew "Radiation" in Engineering Thermodynamics Work and Heat Transfer, 2nd ed., London, Longmans, Green and Co Ltd, 1967, pp 576-577.

[6] M. Fowler "Black Body Radiation". University of Virginia. [Online accessed 07/09/2021]

http://galileo.phys.virginia.edu/classes/252/black_body_radiation.html

[7] D. M. Pozar Microwave Engineering, $2^{\text {nd }}$ ed., John Wiley \& Sons. New York. 1998. pp 608.

[8] A. C. Marvin et al., "A wide-band hybrid antenna for use in reverberation chambers," 2013 IEEE International Symposium on Electromagnetic Compatibility, 2013, pp. 222-226, doi: 10.1109/ISEMC.2013.6670413.

[9] The MathWorks, Inc., MATLAB Function Reference, Accessed on: Oct. 4, 2021. [Online]. Available: https://uk.mathworks.com/help/releases/R2021a/matlab/ref/corrcoef.ht ml?s tid=doc ta\#bunkanr

[10] The MathWorks, Inc., MATLAB Function Reference, Accessed on: Dec. 6, 2021. [Online]. Available: https://uk.mathworks.com/help/releases/R2021a/stats/kstest2.html 